\title{
In-Situ Investigation of Phase Transitions in Functional Poly-Vinylidene Fluoride
}

Suman Kumari ${ }^{1}$, Tugba Isik $^{2}$, Ceren Yilmaz Akkaya ${ }^{3}$ and Volkan Ortalan ${ }^{4}$

${ }^{1}$ Department of Materials Science and Engineering, University of Connecticut, United States, ${ }^{2}$ School of Mechanical Engineering, Purdue University, United States, ${ }^{3}$ Department of Materials Science and Engineering, University of Connecticut, Storrs, Connecticut, United States, ${ }^{4}$ Department of Materials Science and Engineering, University of Connecticut, United States

Polyvinylidene fluoride (PVDF) is a well-known material to be considered in piezoelectric applications. Although there are existing piezoelectric materials such as BaTiO3, PZT, and $\mathrm{ZnO}$, they are limited in their applications due to their brittle nature. PVDF offers advantages over these materials as it is flexible, non-toxic, tough, and resistant to creep.1,2 However, the functional properties of PVDF are bounded by its crystalline polymorphs. Its four major polymorphs are $\alpha, \beta, \gamma$, and $\delta$. Amongst these, $\alpha$ and $\beta$ are the most studied phases because $\alpha$ is the commonly occurring non-polar phase and $\beta$ is the desired polar piezoelectric phase, as shown in Fig $1(\mathrm{a}, \mathrm{b})$. The $\alpha \rightarrow \beta$ transition (with intermediate $\delta$-form) occurs via rotation of dipole molecules in the presence of an external stimulus. 3 This transition was studied heavily in the past to find efficient ways for converting the $\alpha$ phase to a more functional $\beta$ phase. The fraction of $\beta$-phase has been reported to enhance via varying temperature, electric field, solution concentration, mechanical deformation, and evaporation rate.1,4 Despite the number of studies done to improve $\beta$-phase, so far, the phase transformation mechanism has not been studied with in-situ techniques at nanoscale leaving a gap in the fundamental knowledge that can be filled by in-situ transmission electron microscopy methods.

The state-of-the-art transmission electron microscopes (TEMs) can characterize materials at the atomic scale. Besides that, the in-situ heating holders have made it possible to observe the ongoing reaction/transformation in real-time. However, the conventional furnace-type heating holders have limitations due to their low thermal stability.5,6 On the other hand, microelectromechanical systems (MEMS) based TEM specimen holders provide accurate control over in-situ stimuli with high stability, thus maintaining high spatial resolution.6 In addition to controlled heating, MEMS-type chips can apply a high in-situ electric field on micro/nanoscale, which can act as a stimulus for materials transformation. This study focuses on using the MEMS-based chips to apply in-situ heating and electric field to thin PVDF films to initiate phase transformation. In order to increase the electric field stimulus and achieve the high amplitudes required for the phase transition, the distance between the electrodes of the chips needs to be adjusted. We modified this distance by depositing platinum wires and plates on the MEMS chips with the help of a focused ion beam, as shown in Fig 1(d). The distance between the platinum plates can be easily varied to reach the required electric field values for different experiments. In this presentation, the dynamics of $\alpha$ to $\beta$ phase transition of PVDF via annealing and biasing at nanoscale to improve fundamental understanding of the phase transformation processes will be discussed.

This work is supported by the Young Investigator Program of Department of Defense Office of Naval Research (CBET-1437219). 


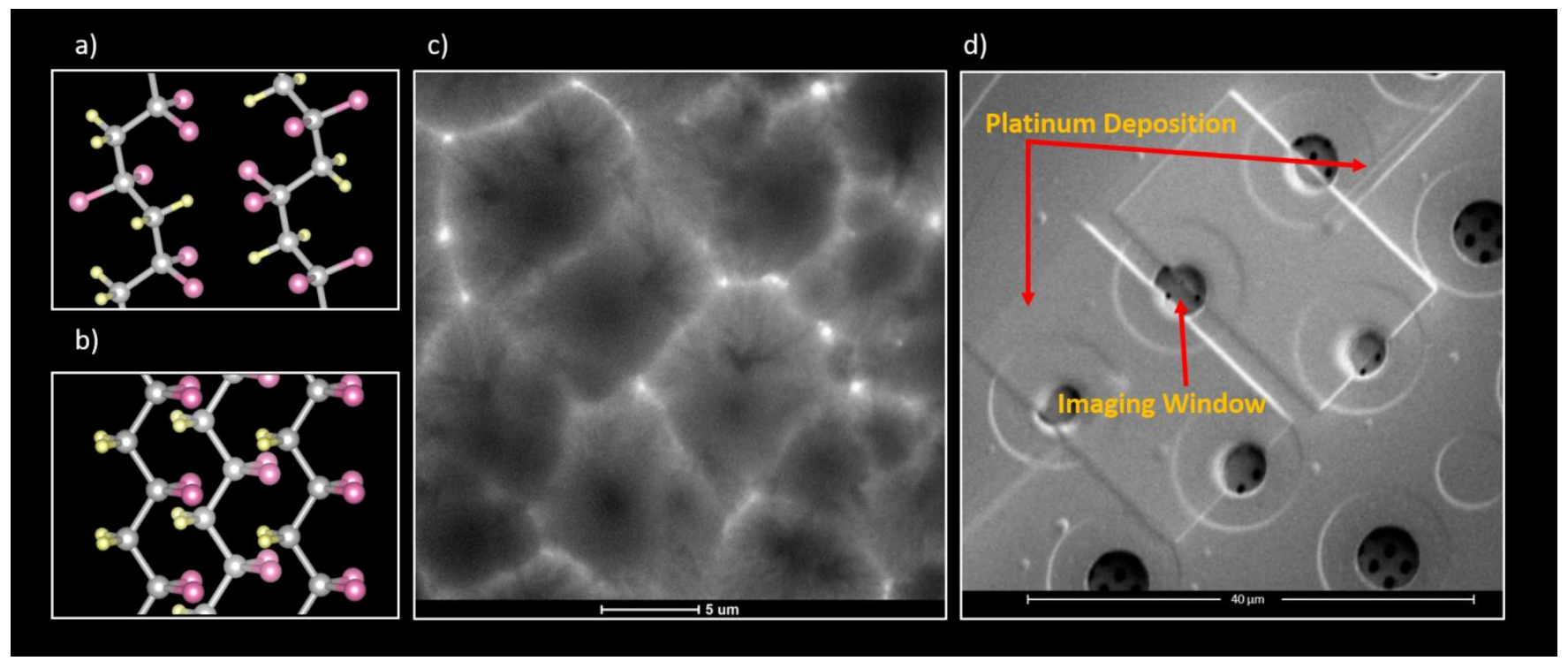

Figure 1. (a) Non-polar $\alpha$-phase and (b) polar $\beta$-phase of PVDF, (c) Spherulite morphology of PVDF, (d) Pt plates and wires deposition on MEMS-type chips using a focused ion beam

\section{References}

1. Shaik, H. et al. Towards $\beta$-phase formation probability in spin coated PVDF thin films. Journal of Polymer Research vol. 24 (2017).

2. Riosbaas, M. T., Loh, K. J., O’Bryan, G. \& Loyola, B. R. In situ phase change characterization of PVDF thin films using Raman spectroscopy. Sensors Smart Struct. Technol. Civil, Mech. Aerosp. Syst. 2014 9061, 90610Z (2014).

3. Ye, Y., Jiang, Y., Wu, Z. \& Zeng, H. Phase transitions of poly(vinylidene fluoride) under electric fields. Integr. Ferroelectr. 80, 245-251 (2006).

4. Horibe, H. et al. Quantification of the solvent evaporation rate during the production of three PVDF crystalline structure types by solvent casting. Polym. J. 46, 104-110 (2014).

5. Vijayan, S. In Situ Investigation of Thermally Activated Processes Using MEMS-Based Devices : Practical Challenges \& Applications. (2018).6. Mele, L. et al. A MEMS-based heating holder for the direct imaging of simultaneous in-situ heating and biasing experiments in scanning/transmission electron microscopes. Microsc. Res. Tech. 79, 239-250 (2016). 